\title{
Impedance variations in Controlled Reactance Parasitic Antennas
}

David V. Thiel, Centre for Wireless Monitoring and Applications.

Griffith University, Nathan, Qld, Australia.d.thiel@griffith.edu.au

Harrington [1] proposed a circular array of reactive loaded parasitic elements surrounding a half-wave dipole antenna. The radiation characteristics are electronically controlled by changing the values of the reactances. Recently this approach to adaptive beam forming applied to a monopole array on a finite ground plane has been of significant interest [2-4]. While some attempts have been made to optimize the structures for maximum gain, often the input impedance of the antenna is not considered. Given that some applications of smart antennas relate to target tracking and null positioning [5], the effect of changing parasitic element reactance on both gain and antenna impedance needs to be assessed simultaneously if the signal direction is to be determined from maximum signal input.

\section{Formulation}

In an array of $N+1$ elements with one active antenna and $N$ parasitic elements without loading, the currents in each element I can be calculated by inverting the equation:

$$
\mathbf{V}=\mathbf{Z I}
$$

where $\mathrm{V}$ is the $1 \mathrm{x} N$ voltage vector with one non-zero element, $\mathrm{I}$ is the $1 \mathrm{x} N$ current vector and $\mathrm{Z}$ is the $N \mathrm{x} N$ mutual impedance matrix [5].

If $N=2$ and the two elements have the same length and radius, and the parasitic element is loaded with impedance $Z_{1}$, then $\mathbf{Z}$ becomes

$$
\mathbf{Z}=\left[\begin{array}{cc}
Z_{11} & Z_{12} \\
Z_{12} & Z_{11}+Z_{1}
\end{array}\right]
$$

where $Z_{11}$ is the self impedance of the element (depends on element length and radius), and $Z_{12}$ is the mutual impedance between the 
elements (depends on element spacing). These elements of $\mathbf{Z}$ can be calculated using the Baker-LaGrone algorithm [6].

The input impedance $Z_{\text {in }}$ of the antenna is calculated from

$$
Z_{\text {in }}=\frac{V}{I_{1}}
$$

where $V$ is the applied voltage and $I_{1}$ is the current in the driven element calculated from (1). Equation (2) can be generalized if the array has additional parasitic elements symmetrically located around the central feed element. These equations have been solved to investigate the change in input impedance as a function of parasitic reactive load for a variety of published antennas.

\section{Results - Two element array}

A variety of reactance ranges have been suggested in the literature. The variation of parasitic load reactance required for optimum antenna gain was quoted from $-401 \mathrm{ohms}$ to $+180 \mathrm{ohms}$ for a seven element circular dipole array with a $\lambda / 4$ element spacing and element diameter of $\lambda / 100$ [1]. In [3] the reactance values were constrained to the range -300 ohms to $300 \mathrm{ohms}$ for a monopole array with the same dimensions. Numerical investigated focused on these ranges.

Figure 1 shows the change in antenna impedance $Z_{\text {in }}$ plotted as a function of load reactance for a two element dipole array with a $\lambda / 4$ spacing between the two elements using equations 1-3. Similar variations are observed using NEC modeling. It can be seen that little effect is likely when the reactance values exceed \pm 300 ohms. This is because the impedance values outside this range present as an open circuit to the antennas and have little effect in beam control [4]. However, very small changes in reactance values close to zero have significant effect on both the input impedance of the antenna and the overall gain of the array.

\section{Results - Seven element array}

Returning to the seven element antennas, the reactance loads suggested by Harrington [1] for ten-degree main beam angular control were modeled using equations (1-3). The input impedance of the antenna changes by less than 5 percent. 
If all loads are identical, then the variation in input impedance of the antenna varies significantly as a function of load reactance (see Figure 2 ). The real part of the impedance varies from approximately $4 \mathrm{ohms}$ to almost $200 \mathrm{ohms}$, and the reactive component of the input impedance varies from 50 to $230 \mathrm{ohms}$ over the same range. The very low real part of the impedance occurs when the loads present close to a short circuit in the parasitic elements. If one parasitic element is assigned a much higher reactive load (either positive or negative), very little change is observed in the input impedance of the antenna. When two or more parasitic elements have a high impedance loading - approximating an open circuit, then the input impedance of the antenna approaches that for an isolated dipole.

The reactance conditions causing these major variations in input impedance place constraints on the beam forming algorithm that might be used for signal tracking or precise angle of arrival determination. In particular, during a null forming procedure, the matching of the antenna to the transmission line can be significantly affected with the net result of a significant reduction in the desired signal as well as the interference source. Even minor variations in received signal strength will occur as a result of changing antenna input impedance rather than imperfect beam alignment. The problem can only be avoided if symmetrical reactance loads are always used as the antenna beam is controlled and the antenna has been matched appropriately.

\section{References:}

[1] R.F. Harrington, "Reactively controlled directive arrays," IEEE Trans. Antennas and Propagation, vol. 26, pp. 390-395, 1978.

[2] T. Ohira and K. Gyoda, "Hand-held microwave direction-of-arrival finder based on varactor-tuned analog aerial beamforming," IEEE Asia Pacific Conference (Taipei), vol. 2, pp. 585-588, Dec. 2001.

[3] R. Schlub, J. Lu, and T. Ohira, "Seven-element ground skirt monopole ESPAR antenna design from a genetic algorithm and finite element method," IEEE Trans. Antennas and Propagation, vol. 51, pp. 3033-3039, 2003.

[4] D.V. Thiel, and S. Smith, Switched parasitic antennas for cellular communications, Artech House, Boston, MA, 2002.

[5] D.V. Thiel, "Switched parasitic antennas and controlled reactance parasitic antennas: A systems approach," IEEE AP Symposium, Monterey CA, vol. 3, pp. 3211-3214, June 2004.

[6] H.C. Baker and A.H. LaGrone, "Digital computation of the mutual impedance between thin dipoles," IRE Trans. Antennas \& Propagation, vol. 10, pp. 172-178, 1962. 


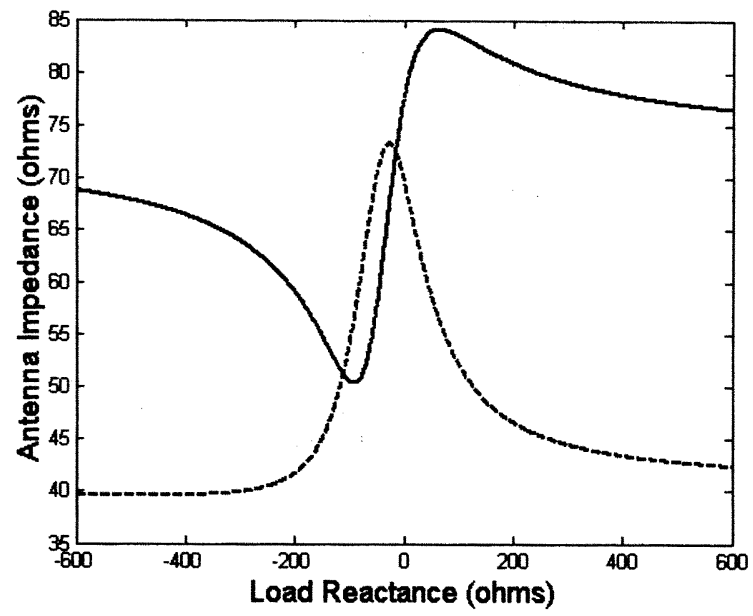

Fig 1: Variation in input impedance (real part continuous line, imaginary part broken line) of a two-element reactance loaded parasitic antenna array.

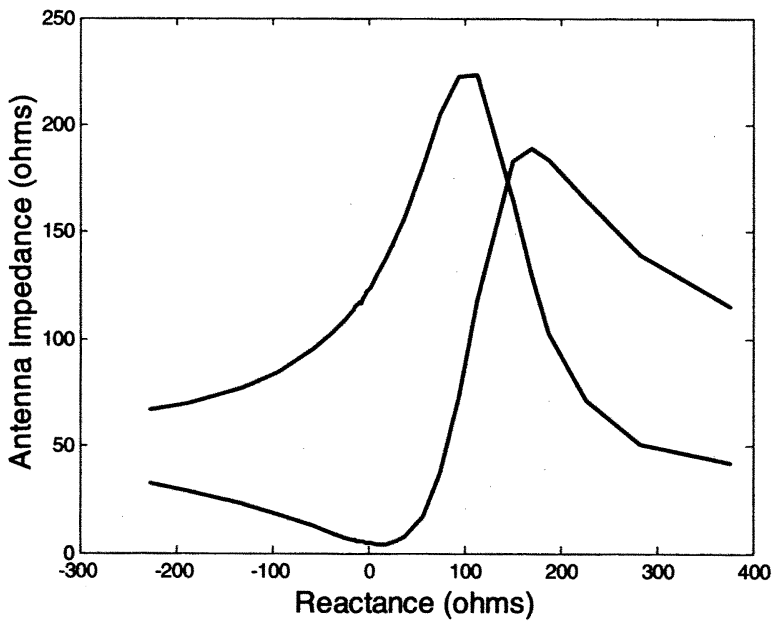

Figure 2: Antenna impedance of seven element dipole controlled reactance array as a function of load reactance with all loadings identical (real part is continuous line, imaginary part is broken line). 